\title{
Article \\ Diffusion-Weighted MRI and Urinary Activin-A are Correlated with the Degree of Hypoxic Ischemic Encephalopathy in Neonates
}

\author{
Sheren E. Maher 1, Mohamed E. Abdelazim 1,*, Mohamed G. Eissawy ${ }^{2}$, Mahmoud M. Higazi ${ }^{2}$ \\ and Nagwa I. Okaily ${ }^{3}$ \\ 1 Pediatric Department, El-Minya University, Minya 11432, Egypt; sherenesammaher@yahoo.com \\ 2 Radiology Department, El-Minya University, Minya 11432, Egypt; mohamedessawy@gmail.com (M.G.E.); \\ mahmodhegazi@gmail.com (M.M.H.) \\ 3 Clinical-Pathology Department, El-Minya University, Minya 11432, Egypt; nagwalab1980@gmail.com \\ * Correspondence: abdelazeemhemed@gmail.com
}

\begin{abstract}
Background: Early identification and prevention of hypoxic ischemic encephalopathy (HIE) may reduce neonatal mortality and morbidity. Objective: We aimed to correlate between urinary Activin-A and MRI (conventional and Diffusion-weighted) and the severity of HIE in fullterm neonates. Methods: Forty-five full-term neonates with HIE admitted to NICU and 15 normal neonates were enrolled into the study. The concentration of urinary Activin-A was determined using enzyme immunoassay kits and MRI were done. Correlations between urinary Activin-A and MRI with the degree of HIE were done. Results: Urinary Activin-A levels were significantly higher in neonates with HIE than controls $(\mathrm{P}<0.001)$. It was positively correlated with the clinical grading of HIE and a cutoff value of $0.08 \mu \mathrm{g} / \mathrm{l}$ on day- 1 after birth had a sensitivity of $98.6 \%$ and specificity of $97.1 \%$ for prediction of HIE. DW-MRI detected HIE with a high sensitivity $(85 \%)$ compared to the low sensitivity of conventional MRI (35\%). An ADC value of $\leq 0.8$ was the best sensitivity-specificity cutoff point for detecting severe ischemic injury. DW-MRI imaging was positively correlated with Urinary Activin-A and both of them were positively correlated with the clinical grades of $\mathrm{HIE}$ ( $\mathrm{P}<$ 0.001). Conclusions: DW-MRI imaging is correlated well with urinary Activin-A in full-term neonates with HIE and both of them are correlated with the degree of HIE. Early determination of urinary Activin-A combined with DW-MRI imaging can early detect HIE and its severity in fullterm neonates with HIE.
\end{abstract}

Keywords: Activin-A; HIE; MRI; apparent diffusion coefficient; neonates

\section{Introduction}

Hypoxic-ischemic encephalopathy (HIE) is an important cause of morbidity and mortality in the neonates especially in developing countries ${ }^{1}$. It can cause severe neurologic disabilities due to its serious complications, such as cerebral palsy and mental retardation ${ }^{2}$. Its prevalence increases as the level of development decreases in a population ${ }^{3}$. Early and accurate diagnosis is helpful not only for assessing prognosis but also for making treatment. Early diagnosis is extremely important for minimizing or even preventing permanent brain damage.

Diffusion-weighted MRI has been shown to be generally sensitive to early ischemic brain changes ${ }^{4}$. Generation of apparent diffusion coefficient (ADC) maps and calculating ADC values allow obtaining an objective measure of diffusion abnormality ${ }^{5}$.

Activin-A is a glycoprotein expressed in CNS and its concentrations increased in blood after ischemic brain injury ${ }^{6}$ and many studies reported that urinary Activin-A is an early marker for HIE in neonates ${ }^{7,8}$. 
In this study, we measured urinary Activin-A immediately as well as MRI (Conventional and DW) and correlated between them and the degree of severity of HIE to predict the occurrence and improve the accuracy of early diagnosis as well as prognosis of HIE.

\section{Patients and Methods}

The study included 60 full term neonates (gestational age ranged from 37 to 42 weeks) selected from delivery room and NICU, Minia University hospital for children during the period from May 2014 to July 2016. They were divided into 4 groups: Group I: asphyxiated neonates with mild HIE included 13 neonates ( 7 females and 5 males), group II: asphyxiated neonates with moderate HIE and at some risk of neurological handicap, included 16 neonates ( 7 females and 8 males), group III: asphyxiated neonates with severe HIE and at a greater risk of major neurological handicap, included 16 neonates (10 females and 8 males)and group IV: control group, included 15 apparently healthy full term neonates ( 8 females and 7 males).

\subsection{Selection of Patients}

Inclusion criteria: HIE was defined as the presence of 3 or more of the following: Profound metabolic acidosis ( $\mathrm{pH}$ less than 7.00 and base deficit $\leq-12 \mathrm{mmol} / \mathrm{L}$ ) on an umbilical cord arterial blood sample, early onset of severe or moderate neonatal encephalopathy in infants born at 37 or more weeks of gestation with neurological dysfunction including altered level of consciousness and reactivity, altered tone, hyperactive or absent reflexes, weak or absent Moro reflex and seizures, sentinel hypoxic event during labor (heart rate of less than 100 beats per minute, late decelerations, or reduced beat-to-beat variability or, severe electronic fetal monitoring abnormalities, thick meconium stained amniotic fluid and respiratory depression, hypotonia, or bradycardia, a need for resuscitation for more than 3 minutes with positive pressure ventilation and oxygen immediately after birth , Apgar score of 0 to 3 beyond five minutes, onset of multisystem involvement within 72 hours of birth.

\subsection{Exclusion Criteria}

Detectable congenital anomalies or fetal malformations, congenital perinatal infections including chorio-amnionitis, maternal diabetes or hypertension, intrauterine growth restriction, prematurity $<37$ weeks gestation, multiple pregnancies, coagulation disorders, suspected cardiac, metabolic, renal or hepatic cell failure or hemolytic diseases.

Asphyxiated neonates with HIE diagnosed and graded according to Sarnat and Sarnat staging system ${ }^{9}$. Asphyxiated neonates with moderate or severe HIE were identified when there is neurological dysfunction including altered level of consciousness and reactivity, altered tone, hyperactive or absent reflexes, weak or absent Moro reflex and seizures.

Neonates of the four groups were subjected to thorough antenatal and natal history, physical examinations, including neurological examinations, were performed using the Prechtl test to classify neonatal neurological conditions ${ }^{10}$, assigning each infant to 1 of 3 diagnostic groups, normal, suspect, or abnormal, in accordance with Jurgens-van der Zee et al ${ }^{11}$. Standard cerebral ultrasound (Acuson 128SP5) was performed for patients and controls ${ }^{12}$. Electroencephalogram (EEG) was performed for all asphyxiated neonates. Cord blood gases, $\mathrm{CBC}$, blood group ( $\mathrm{Rh}$ and $\mathrm{ABO}$ of parents and neonates) and total serum bilirubin were done.

Activin-A was measured at first urination (within the first 24 hours), at 48 hours and at 72 hours of age. The mean collection times on days $1-3$ were $6 \pm 1.5,37 \pm 2.4$ and $61 \pm 2.4$ hours after birth, respectively. Urine samples were collected with a standard urine collector (Serenity, Artsana S.p.A) at the indicated time points. At each time point, $5 \mathrm{~mL}$ urine samples collected in the previous hour were immediately centrifuged at $900 \mathrm{~g}$ for $10 \mathrm{~min}$, and supernatants were stored at $-70^{\circ} \mathrm{C}$. Activin-A measurements were performed in with a 2-site enzyme immunoassay kits from Serotec. The detection limit (limit of the blank) was $-10 \mathrm{ng} / \mathrm{L}$; intra- and interassay imprecision (CVs) were $2.5 \%$ and $3.0 \%$, respectively. The laboratory investigations were done in clinical pathology department, faculty of Medicine, Minia university hospital for children. 


\subsection{Patient Preparation for MRI}

The neonates were laid in a supine position. Neonatal head coil was used. If necessary, the neonates were sedated. If the neonate was on antiepileptic medication, the dose of antiepileptic medication was increased just before MRI procedure. Those who were not on sedatives or antiepileptic medication received chloral hydrate, $50-70 \mathrm{mg} / \mathrm{kg}$ before imaging.

MRI examination: The patients were examined on $1.5 \mathrm{~T}$ MRI scanner (Philips Achieva). DW images were acquired in an axial plane, TR/TE 5000/90 msec, slice thickness $4 \mathrm{~mm}$ with inter-slice gap of $8 \mathrm{~mm}$ and matrix: 112 x 96 at b values (0, and 1000). DW-MRI visual Analysis: DiffusionWeighted MR Images were assessed for abnormal signal intensity areas at different locations of the brain that could be encountered in HIE; including basal ganglia and thalamus (BGT), posterior limb of internal capsule (PLIC), and parasagittal white matter (WM). ADC measurement: Pixel-based ADC maps were generated on a workstation. A region of interest was drawn over hyper-intensity areas representing restricted diffusion on diffusion images. The corresponding ADC values were measured. The area at each region of interest was $0.3-1 \mathrm{~cm}^{2}$, except in the PLIC, where the region-ofinterest areas were $0.15-0.2 \mathrm{~cm}^{2}$. Care was taken to avoid inclusion of CSF at region of interest.

DW-MRI images were assessed only for forty neonates with HIE out of forty five (as we could not do MRI for five cases) for areas of restricted diffusion at different locations that could be encountered in HIE.

Informed consents were obtained from all parents of infants before inclusion in the study, for which local departmental research committee approval was obtained.

\subsection{Statistical Analysis}

Results of this study were expressed as mean \pm SD or percentage. Comparison between clinical and laboratory parameters of different groups were done by $t$-test and ANOVA test. The specificity, sensitivity, positive predictive value (PPV), and negative predictive values (NPV) of markers were calculated using cut off points for urinary Activin-A. Receiver Operator Characteristic (ROC) curve was constructed. Significant difference was considered when P value $<0.05$.

\section{Results}

Out of 45 full term neonates with HIE, 23 neonates showed normal neurological examination, 18 patients were classified as suspect (11 with hypotonia and 7 with hyper-excitability) and 6 patients have abnormal neurological examination. The results of ultrasonography showed that: eight neonates had diffuse brain edema, five had hyper-echogenicity in the bilateral basal ganglia and thalamus, one had infarctions in the middle cerebral artery, and one had multicystic encephalomalacia. Nine infants in this group had severe diffuse abnormalities on the EEG (a burst-suppression pattern, unreactive traces, or marked voltage suppression) and six had focal epileptiform discharges on EEG.

Gestational age, weight, sex distribution, hemoglobin concentration, reticulocytic count, TLC, total bilirubin, urea and creatinine did not differ at birth between neonates with HIE and controls while mode of delivery , Apgar scores at the 1st and 5th minutes and $\mathrm{pH}$ were significantly different ( $\mathrm{P}=0.04$, 0.001and 0.001respictively) (Table 1).

From day 1 to day 3 the urinary Activin-A concentration was significantly higher in neonates with HIE than those in the controls. Also they were significantly higher in neonates with either moderate or severe HIE than in those with mild HIE $(\mathrm{P}<0.001)$ but there was no significant difference between neonates with moderate HIE and those with severe HIE $(P>0.05)$. So the increase in urinary Activin-A level was positively correlated with the severity of HIE ( $<<0.001)$ (Table 2 ).

MRI images were done for only forty cases out of forty-five as there were five cases did not perform MRI (two cases died before imaging, two cases their parents refused to do MRI and one case was difficult to do MRI for technical problems).

The mean age of neonates at time of MRI imaging was 5 days (range: 3-11 days). Twelve neonates (30\%) were imaged early within the first week postnatal while $28(70 \%)$ neonates were imaged late after 7 days. 
Table 1. Clinical and laboratory data of studied groups.

\begin{tabular}{cccccc}
\hline Item & $\begin{array}{c}\text { Group I } \\
\text { (n=13) }\end{array}$ & $\begin{array}{c}\text { Group II } \\
\text { (n=16) }\end{array}$ & $\begin{array}{c}\text { Group III } \\
(\mathbf{n = 1 6})\end{array}$ & $\begin{array}{c}\text { Group IV } \\
\text { (n=15) }\end{array}$ & P-value \\
\hline Gestational age (weeks) & $37.8 \pm 2.2$ & $37.3 \pm 2.5$ & $37.5 \pm 2.7$ & $37.2 \pm 2.8$ & 0.14 \\
Birth weight (Kg) & $3.6 \pm 1.2$ & $3.4 \pm 1.7$ & $3.8 \pm 1.8$ & $3.5 \pm 1.9$ & 0.13 \\
Sex: Male & $7(53.8 \%)$ & $8(50 \%)$ & $10(62.5 \%)$ & $8(53.3 \%)$ & 0.19 \\
Female & $6(46.1 \%)$ & $8(50 \%)$ & $6(37.5 \%)$ & $7(46.7 \%)$ & \\
Mode of delivery: SVD & $4(30.7 \%)$ & $3(18.7 \%)$ & $4(25 \%)$ & $9(60 \%)$ & $0.04^{*}$ \\
& $9(69.3 \%)$ & $13(81.2 \%)$ & $12(75 \%)$ & $6(40 \%)$ & \\
CS & & & & & \\
Apgar score: At 1 minute & $3.7 \pm 0.17$ & $2.8 \pm 0.16$ & $2.4 \pm 0.15$ & $7.9 \pm 1.16$ & $0.001^{* *}$ \\
At 5 & $4.6 \pm 0.27$ & $3.5 \pm 0.25$ & $3.2 \pm 0.24$ & $8.6 \pm 0.23$ & $0.001^{* *}$ \\
minute & & & & & \\
Hb concentration (gm/dl) & $12.9 \pm 5.18$ & $11.3 \pm 5.13$ & $10.4 \pm 5.11$ & $13.3 \pm 4.19$ & 0.32 \\
TLCs (x103/ul ) & $7.3 \pm 1.45$ & $6.2 \pm 1.25$ & $6.3 \pm 1.42$ & $5.35 \pm 1.40$ & 0.08 \\
Reticulocytes (\%) & $2.2 \pm 1.16$ & $3.1 \pm 1.18$ & $2.3 \pm 1.27$ & $2.0 \pm 1.18$ & 0.1 \\
Total biluribin (mg/dl) & $1.7 \pm 0.14$ & $1.9 \pm 0.16$ & $2.2 \pm 0.15$ & $1 \pm 0.15$ & 0.1 \\
Arterial cord blood pH & $6.3 \pm 0.15$ & $6.4 \pm 0.25$ & $6.3 \pm 0.22$ & $7.34 \pm 0.11$ & $0.001^{* *}$ \\
Urea (mg/dL) & $39.2 \pm 15.18$ & $40.6 \pm 10.16$ & $41.4 \pm 12.16$ & $37.2 \pm 9.18$ & 0.26 \\
Creatinine (mg/dL) & $0.77 \pm 0.24$ & $0.82 \pm 0.35$ & $0.87 \pm 0.14$ & $0.81 \pm 0.13$ & 0.73 \\
\hline
\end{tabular}

*Significant $\quad$ ** highly significant

CS: Cesarean section. Hb: Hemoglobin, SVD: Spontaneous vaginal delivery, TLC: Total leucocytic count

Table 2. Urinary Activin-A in patients and controls.

\begin{tabular}{|l|l|l|l|l|}
\hline \multirow{2}{*}{ Group } & \multirow{2}{*}{ No. } & \multicolumn{3}{|l|}{ Urinary Activin-A $(\mu \mathrm{g} / \mathrm{L})$} \\
\cline { 3 - 5 } & & First 24 hours & At 48 hours & At 72 hours \\
\hline Total HIE & 45 & $0.15 \pm 0.07$ & $0.12 \pm 0.06$ & $0.1 \pm 0.05$ \\
\hline Severe HIE & 16 & $0.22 \pm 0.01$ & $0.2 \pm 0.02$ & $0.19 \pm 0.01$ \\
\hline Moderate HIE & 16 & $0.19 \pm 0.08$ & $0.17 \pm 0.09$ & $0.16 \pm 0.08$ \\
\hline Mild HIE & 13 & $0.05 \pm 0.04$ & $0.04 \pm 0.03$ & $0.03 \pm 0.02$ \\
\hline Control group & 15 & $0.01 \pm 0.004$ & $0.01 \pm 0.002$ & $0.013 \pm 0.003$ \\
\hline P - value & $0.001^{* *}$ & $0.001^{* *}$ & $0.001^{* *}$ \\
\hline \multicolumn{5}{|c|}{ Highly significant } \\
\hline
\end{tabular}

Table 3. The mean $\pm \mathrm{SD}$, median, range and $25-75 \%$ of ADC values at different regions of the brain.

\begin{tabular}{ccccc}
\hline Item & Mean \pm SD & Median & Range & 25 - 75 \% of ADC \\
\hline Lentiform & $1.021 \pm 0.208$ & 1.020 & $0.654-1.460$ & 0.969 to 1.155 \\
Thalamus & $0.997 \pm 0.247$ & 1.025 & $0.579-1.450$ & 0.838 to 1.125 \\
PLIC & $0.939 \pm 0.225$ & 0.935 & $0.617-1.480$ & 0.772 to 1.060 \\
PV & $1.037 \pm 0.212$ & 1.040 & $0.653-1.138$ & 0.967 to 1.055 \\
\hline
\end{tabular}

PLIC: posterior limb internal capsule, PV: Periventricular.

Table 4. Diagnostic values of Urinary Activin-A in neonates with HIE.

\begin{tabular}{ccccc}
\hline U. Activin-A & Sensitivity (\%) & Specificity (\%) & PPV (\%) & NPV (\%) \\
\hline At day 1 & 98.2 & 97.1 & 97.2 & 100 \\
At day 2 & 91.4 & 92.9 & 95.3 & 100 \\
At day 3 & 81.3 & 81.1 & 82.0 & 81.4 \\
\hline
\end{tabular}

PPV: positive predictive value, NPV: negative predictive value. 
Conventional imaging: The full extent of ischemic injury was demonstrated by conventional MRI imaging (T1WI \& T2WI) in $8(20 \%)$ cases and detected part of the injury in $6(15 \%)$ cases with a sensitivity of (15\%). The visual analysis of DWI: Ischemic damage was detected on diffusionweighted images and confirmed by a reduced ADC in $26(65 \%)$ patients with an overall sensitivity of about (85\%). Diffusion weighted imaging showed normal findings in $14(35 \%)$ neonates. It detected part of the injury in $18(45 \%)$ subjects with a sensitivity of about (45\%). Eight (20\%) injuries were equally well detected by conventional MR imaging, diffusion-weighted imaging and ADC. Twentyfour $(60 \%)$ cases had focal patchy restriction while two cases $(5 \%)$ had diffuse restriction. Summary of ADC values detected at different locations of injured brain showed no significant differences at different regions of involved brain tissues ( $p$ values $>0.05$, ANOVA test) (Figure $1 \& 3$ ).

Receiver operator characteristics (ROC) curve for capability of ADC values of discriminating $\mathrm{mild} /$ moderate from severe brain tissue injury showed high area under curve (AUC $=0.979,95 \% \mathrm{CI}$ 0.735 to 1.000 ) with $\mathrm{p}$-value $<0.001$ indicating good capability. An ADC value of $\leq 0.8$ was detected as best sensitivity specificity cut off point; as it achieved $85 \%$ sensitivity and $100 \%$ specificity for detecting severe ischemic brain injury at any location. (Figure 2).

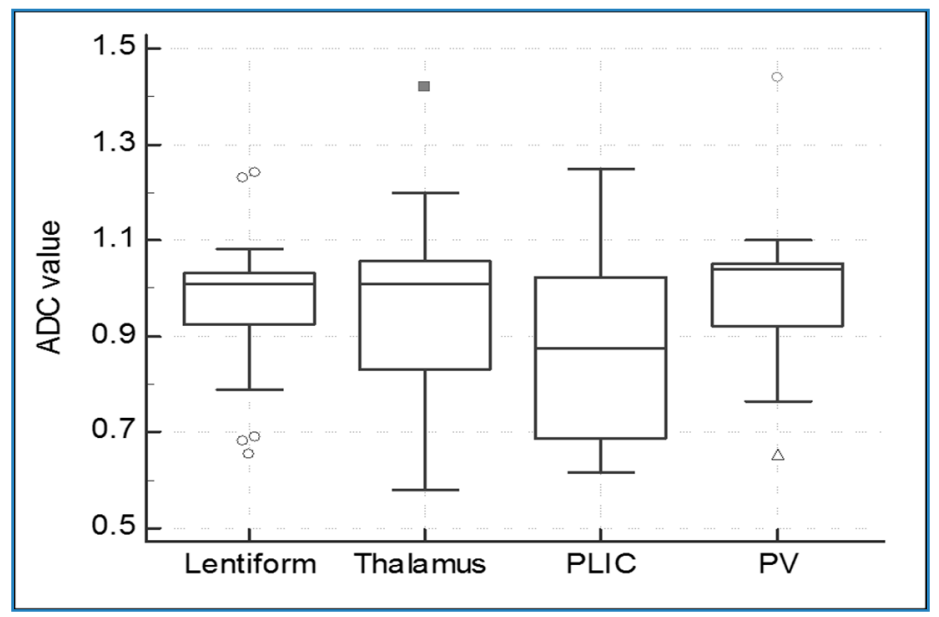

Figure 1. Shows ADC values at different locations of injured brain tissues.

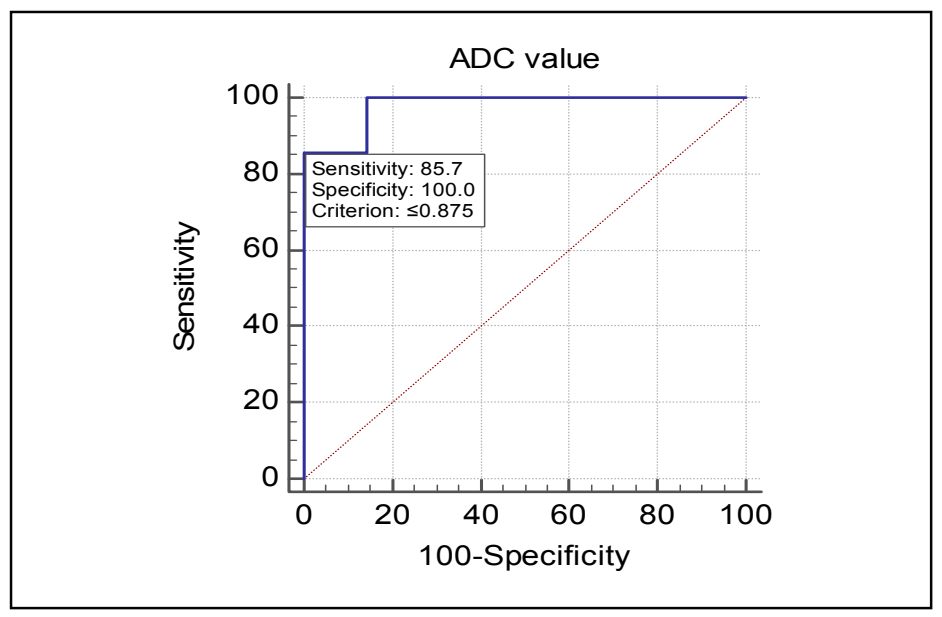

Figure 2. (ROC) curve for capability of ADC values of discriminating mild/moderate from severe brain tissue injury. 


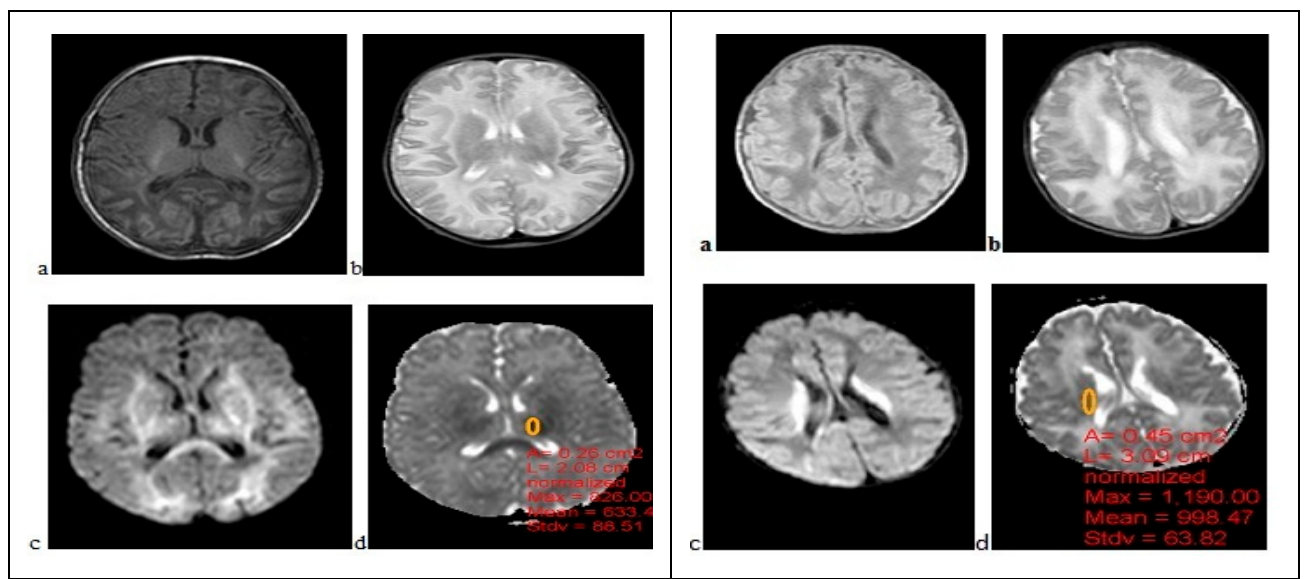

Figure 3. Left $(a, b)$ Conventional MRI: showing normal findings while (c,d) DW-MRI: showing restricted diffusion at periventricular area with low ADC values (0.998). Right (a, b) Conventional MRI showing normal findings while (c, d) DW-MRI: showing diffusion restriction at BGT \& PLIC with very low ADC values (0.633).

\section{Discussion}

Global estimates for asphyxia-related neonatal deaths vary from 0.7 to 1.2 million annually ${ }^{13}$. Peri-partum asphyxia remains an important cause of long-term sensori-neural impairments and disabilities ${ }^{14}$.

Clinical confirmation of ischemic damage is often difficult at this time, when the neonate may present with nonspecific clinical features. In addition, clinical evaluation alone is often inadequate to provide an accurate prognosis.

During the last 2 decades, evidence from experimental and clinical studies proved that therapeutic hypothermia reduces cerebral injury and improves neurological outcome provided that cases of HIE are early diagnosed ${ }^{15-17}$. Many studies of perinatal asphyxia have measured several biologic markers (brain-specific creatine kinase, neurone specific enolase, S $100 \mathrm{~B}$ protein, Activin-A, hypoxanthine, erythropoietin, and lactate dehydrogenase in serum or cerebrospinal fluid), but the tests are usually performed late after birth, when the infants may already have HIE ${ }^{18-21}$. These tests may be useful as markers of tissue injury, but they offer little information that can be used to identify neonate infants at high risk for HIE. Based on these studies we studied the urinary Activin-A as an early marker for HIE and MRI were done to correlate between them and the severity of HIE injury.

The low molecular weight of Activin-A (around 28-kDa) allowed it to be excreted in urine through a passive mechanism, and renal function appeared to be normal or at least not different among the groups in this study. Because Activin- $\mathrm{A}$ is of neuronal origin ${ }^{6}$, the present data support the hypothesis that damage to the brain from hypoxia/asphyxia increases Activin-A in urine. These findings are consistent with the evidence of increased Activin-A concentrations in neonates with perinatal hypoxia and ischemic injury 7,8 . The protein half-life of Activin-A is estimated to be around one hour in the circulation, so the persistently high urine concentrations of Activin-A in asphyxiated were likely attributable to the continuous release of it from damaged nervous tissue ${ }^{21}$.

In this study, we selected our neonates with HIE and followed them to determine the severity of HIE. Infants who had severe HIE were hypotonic, inactive, or comatose within six hours after birth, the infants who would later have mild HIE were not easily distinguishable from those in whom HIE did not develop. Some infants with low Apgar scores have signs of progressive neurologic dysfunction, including an initial period of near normality followed by seizures, deterioration of muscle tone, and deepening coma. A moderate brain insult can be difficult to detect when other conditions, such as meconium aspiration syndrome, are present or when sedative or paralytic drugs have been administered ${ }^{22}$.

Electroencephalographic changes can be valuable in identifying infants at high risk for subsequent brain damage, but the interpretation of neonatal EEG can vary and requires considerable experience ${ }^{23}$. 
In the present study, at different detection time, urinary Activin-A levels were higher in neonates with HIE than those in the control group, and their urinary levels were significantly higher in those with moderate and severe HIE than in those with mild HIE and the control group. The changes of urinary Activin-A levels were basically consistent with the clinical grades, i.e., the more severe the brain damage, the higher the urinary Activin-A level.

This indicated that urinary Activin-A was positively correlated with brain damage. It is possibly because fewer neurons of the neonates with mild HIE suffered from necrosis, thus no significant changes were observed in urinary Activin-A levels; whereas in neonates with severe HIE, marked increase in urinary Activin-A level was found due to the heavy involvement of the brain, as reported previously ${ }^{21-23}$.

Therefore, it is feasible that urinary Activin-A level can be used as a sensitive and reliable index for early identification of HIE brain damage as well as for assessment of the severity of the damage. These results are in agreement with Pasquale et al. ${ }^{21}$ who stated that urinary Activin-A was already higher in those infants with adverse neurological outcome at a stage when ultrasound and other diagnostic procedures did not predict outcome. These findings may also provide support for repeated monitoring of Activin-A in urine to evaluate the efficacy of therapeutic measures.

MRI changes due to hypoxic-ischemic cerebral damage at conventional MRI scans are often subtle and may be difficult to interpret or to distinguish from normal (maturational) phenomena in neonatal brain ${ }^{24}$. This is in contrast to high signal to noise ratio at images depicted by DW-MRI (bright ischemic tissue against dark background). Diffusion-weighted imaging (DWI) is a sensitive imaging modality to detect ischemic changes, in earliest phase; depending on early restricted diffusion within ischemic brain tissue ${ }^{25}$. In addition, it provides quantitative apparent diffusion coefficient (ADC) values within brain tissue ${ }^{26}$.

The clinical manifestations and MRI imaging patterns of HIE in term infants depend upon duration and severity of the hypoxic or ischemic insult. The peripheral pattern occurs in the setting of mild to moderate hypotension or partial hypoxia with a prolonged duration ${ }^{27}$. The basal gangliathalamus pattern usually occurs in the setting of a severe hypoxic or profound hypotensive event with a shorter duration but in many cases these patterns may often overlap ${ }^{28}$.

In this study, the 5 minutes Apgar scores were well correlated with MRI severity and these results were in agreement with other reports had shown that pattern of injury differed between low \& high APGAR scores. Most of their patients (70\%) with low Apgar scores had basal ganglia thalamus (BGT) lesions. The association between low Apgar scores and BGT lesions is not surprising as BGT lesions are known to be associated with acute severe hypoxic insults ${ }^{29}$.

In this study, abnormal diffusion by DW-MRI was seen in 34 cases out of 40 neonates who were involved; with an overall sensitivity of $(85 \%)$. In the meantime, only 14 injuries were detected by conventional imaging with an overall sensitivity of (35\%). The full extent of the ischemic encephalopathy was demonstrated by conventional MRI (T1WI \& T2WI) in 10 cases (25\%) only. The low sensitivity of conventional MRI can be explained by high water content of the brain at this early age; with subsequent normal conventional MRI despite significant brain encephalopathy, especially when imaging is performed early ${ }^{30}$.

DW-MRI can be considered as a preferred modality for imaging of HIE due to its high sensitivity and easy interpretation. This is in addition to being a fast imaging sequence, with no contrast administration.

Similar to our results, many studies reported that DWI was the most sensitive imaging modality for detecting HIE in its acute period as well as DWI and ADC measurements are helpful in improving detection and depiction of extent of injury in the setting of acute and/or sub-acute HIE H1-33. $^{3}$.

In this study; ADC value of $0.8 \times 10^{-3} \mathrm{~mm}^{2} / \mathrm{sec}$. was detected as a cutoff point between severe and mild/moderate cases. Hunt et al. ${ }^{34}$ found that mean ADC values (among survivors after perinatal asphyxia) were $0.89+/-0.17$ in PLIC and it was also associated with poor motor outcome, whereas mean ADC value (among non-survivors) were $0.75+/-0.17$. Vermuelen et al. ${ }^{30}$ postulated that all infants with an ADC value in the PLIC lower than $85 \times 10^{-5} \mathrm{~mm}^{2} / \mathrm{sec}$ had a poor motor outcome or death.

When we examined the effect of image timing on ADC values, we could not detect any significant differences between neonates who imaged before or after 7 days in our results as well as 
we did not find significant correlation between ADC values and the age of neonates at time of imaging. These results were in agreement with other reports ${ }^{35}$.

On another hand; Rutherford et $\mathrm{al}^{36}$ found normal ADC values in moderate WM and BGT lesions during the first week after hypoxic-ischemic event and this means that normal ADC values did not exclude hypoxic-ischemic brain damage in neonates.

In this study, there were positive significant correlations between urinary Activin-A along the study and ADC as well as between both of them and the degree of severity of HIE so, combination of urinary Activin-A and MRI improve the diagnostic value of MRI for evaluation of HIE and ADC values for DW-MRI should always be interpreted in combination with visual analysis of both conventional and diffusionweighed images and timing of scan needs to be taken into consideration.

From this study, we can conclude that DW-MRI is a preferred modality of imaging of HIE due to its high sensitivity and easy interpretation especially if combined with urinary Activin-A levels.

This study is not without limitations; no follow-up data for long periods were available for studied patients. Adding, encephalopathy patterns may evolve over time, therefore, more than one-time point may need to be assessed, but we did not perform follow-up imaging because of extra burden. We recommend further studies with large simple size to focus more on more biochemical and radiological markers for HIE.

\section{Conclusion}

DW-MRI imaging is correlated well with urinary Activin-A in full-term neonates with HIE and both of them are correlated with the degree of HIE. Early determination of urinary Activin-A combined with DW-MRI imaging can early detect HIE and its severity in neonates. These findings may be helpful in planning strategies for early diagnosis and intervention of HIE.

\section{References}

1. Bhutta ZA, Haider BA. Birth asphyxia in developing countries: Current status and public health implications. Curr Probl Pediatr Adolesc Health Care 2006; 36:178.-88.

2. Allen KA, Brandon DH. Hypoxic Ischemic Encephalopathy: pathophysiology and experimental treatments 2011; 11(3):125-133.

3. Dağ Y, Firat AK, Karakaş HM, et al. Clinical outcomes of neonatal hypoxic ischemic encephalopathy evaluated with diffusion-weighted magnetic resonance imaging 2006; 12:109-114.

4. Agut T, León M, Rebollo M, Muchart J, Arca G, Garcia-Alix A. Early identification of brain Encephalopathy in infants with hypoxic ischemic encephalopathy at high risk for severe impairments: accuracy of MRI performed in the first days of life. BMC Pediatr 2014;14:177.

5. Liauw L., Veen S., van Buchem M.A., et al; Do Apparent Diffusion Coefficient Measurements Predict Outcome in Children with Neonatal Hypoxic-Ischemic Encephalopathy? AJNR Am J Neuroradiol 2009;30:264-70.

6. Luisi S, Florio P, Reis FM, Petraglia F. Expression and secretion of Activin-A: possible physiological and clinical implications. Eur J Endocrinol 2001;145: 225-36.

7. Florio P, Gazzolo D, Luisi S, Petraglia F. Activin in brain injury. Adv Clin Chem 2006; (53) 518-22.

8. Florio P, Perrone S, Luisi S, Longini M, Tanganelli D, Petraglia F, et al. Activin a plasma levels at birth: an index of fetal hypoxia in preterm neonate. Pediatr Res 2003; 54:696-700.

9. Sarnat HB, Sarnat MS. Neonatal encephalopathy following fetal distress. A clinical and electroencephalographic study. Arch Neurol. 1976; 33: 696 -705.

10. Prechtl HFR. Assessment methods for the neonate infant: a critical evaluation. In: Stratton D (ed). Psychobiology of human neonate. Wiley, Chichester 1982;21-52.

11. Jurgens-van der Zee AD, Bierman-van Eendenburg ME, Fidler VJ, Olinga AA, Visch JH, Touwen BC, et al. Preterm birth, growth retardation and acedemia in relation to neurological abnormality of the neonate. Early Hum Dev 1979;3/2:141-54.

12. Barkovich AJ. Brain and spine injuries in infancy and childhood. In: Barkovich AJ, ed. Pediatric Neuroimaging. Philadelphia: Lippincott Williams \& Wilkins; 2005:190-290.

13. Lawn J, Shibuya K, Stein C. No cry at birth: global estimates of intrapartum stillbirths and intrapartumrelated neonatal deaths. Bull World Health Organ. 2005; 83(6):409-417. 
14. Gonzalez FF, Miller SP. Does perinatal asphyxia impair cognitive function without cerebral palsy? Arch Dis Child Fetal Neonatal Ed. 2006;91(6):F454-F459.

15. Cotten CM, Shankaran S. Hypothermia for hypoxic-ischemic encephalopathy. Exp. Rev. Obst. \& Gyn. 2010; 5:227-239

16. Shah PS. Hypothermia: a systematic review and meta-analysis of clinical trials. Semin Fetal Neonatal Med. 2010;15(5):238-246.

17. Azzopardi DV, Strohm B, Edwards AD, et al; TOBY Study Group. Moderate hypothermia to treat perinatal asphyxial encephalopathy. N Engl J Med. 2009; 361(14):1349-1358.

18. Perlman J. Markers of asphyxia and neonatal brain injury. N Engl J Med 1999; 341:363-5.

19. Erdag G, and Vitrinel A. Can Urinary Uric Acid / Creatinine Ratio Be Used as an Additional Marker for Neonatal Asphyxia? International Pediatrics 2004; (19) 217-219

20. Thornberg E, Thiringer K, Hagberg H, Kjellmer I. Neuron specific enolase in asphyxiated neonates: association with encephalopathy and cerebral function monitor trace. Arch Dis Child Fetal Neonatal Ed 1995;72: F39-F42.

21. Pasquale F, Stefano L, Bashir M, et al., High Urinary Concentration of Activin-A in Asphyxiated Full Term Neonates with Moderate or Severe Hypoxic Ischemic Encephalopathy. Clinical Chemistry 2007, 53 (3) 520 5220 .

22. Laing I, Brown JK, Harkness RA. Clinical and biochemical assessments of damage due to perinatal asphyxia: a double blind trial of a quantitative method. J Clin Pathol 1988; 41: 247-52.

23. Vannucci RC. Interventions for perinatal hypoxic-ischemic encephalopathy. Pediatrics 1997;100:1004-14.

24. Alderliesten T, Vries LS de, Benders MJNL, Koopman C, Groenendaal F. MR Imaging and Outcome of Term Neonates with Perinatal Asphyxia: Value of Diffusion-weighted MR Imaging and $H$ MR Spectroscopy. Radiology 2011;261:235-42.

25. Dağ Y, Firat AK, Karakaş HM, Alkan A, Yakinci C, Erdem G. Clinical outcomes of neonatal hypoxic ischemic encephalopathy evaluated with diffusion-weighted magnetic resonance imaging. Diagn Interv Radiol 2006;12:109-14

26. Cavalleri F, Lugli L, Pugliese M, D'Amico R, Todeschini A, Della Casa E, et al. Prognostic value of diffusionweighted imaging summation scores or apparent diffusion coefficient maps in neonates with hypoxicischemic encephalopathy. Pediatr Radiol 2014;44:1141-54. doi:10.1007/s00247-014-2945-9.

27. Rutherford M, Counsell S, Allsop J, et al. Diffusion-weighted magnetic resonance imaging in term perinatal brain injury: a comparison with site of lesion and time from birth. Pediatrics 2004; 114:1004-14.

28. Ghei SK, Zan E, Nathan JE,et al. MR Imaging of Hypoxic-Ischemic Injury in Term Neonates: Pearls and Pitfalls1 RadioGraphics 2014; 34:1047-1061.

29. Mercuri, E. et al. MRI lesions and infants with neonatal encephalopathy. Is the Apgar score predictive? Neuropediatrics, 2002;33(3), pp.150-6.

30. Vermeulen RJ, van Schie PEM, Hendrikx L, Barkhof F, van Weissenbruch M, Knol DL, et al. Diffusionweighted and conventional MR imaging in neonatal hypoxic ischemia: two-year follow-up study. Radiology 2008;249:631-9.

31. Huang BY, Castillo M. Hypoxic-Ischemic Brain Injury: Imaging Findings from Birth to Adulthood 2008; 28:417-439.

32. Soul, J.S. et al. Time course of changes in diffusion-weighted magnetic resonance imaging in a case of neonatal encephalopathy with defined onset and duration of hypoxic-ischemic insult. Pediatrics,2001; 108(5), pp.1211-4.

33. Chilla GS, Tan $\mathrm{CH}, \mathrm{Xu} \mathrm{C}$, et al. Diffusion weighted magnetic resonance imaging and its recent trend-a survey2015; 5(3): 407-422

34. Hunt RW, Neil JJ, Coleman LT, Kean MJ, Inder TE. Apparent diffusion coefficient in the posterior limb of the internal capsule predicts outcome after perinatal asphyxia. Pediatrics 2004; 114:999-1003.

35. Liauw L, der Grond JV, Annette A. et al. Hypoxic-Ischemic Encephalopathy: Diagnostic Value of Conventional MR Imaging Pulse Sequences in Term-born Neonates. RSNA Radiology 2008; (1)247.

36. Rutherford MA and Cowan FM. MRI of the Neonatal Brain: Magnetic resonance imaging of the normal infant brain:term to 2 years: 2002; 234-37

(C) 2017 by the authors. Licensee Preprints, Basel, Switzerland. This article is an open access article distributed under the terms and conditions of the Creative Commons by Attribution (CC-BY) license (http://creativecommons.org/licenses/by/4.0/). 